\title{
Loss of heterozygosis on chromosome 18q21-23 and muscle-invasive bladder cancer natural history
}

\author{
TOMMASO CAI ${ }^{1}$, NICOLA MONDAINI ${ }^{2}$, DANIELE TISCIONE ${ }^{1}$, MAURIZIO DAL CANTO ${ }^{3}$, \\ RAFFAELLA SANTI ${ }^{4}$, RICCARDO BARTOLETTI ${ }^{2}$ and GABRIELLA NESI ${ }^{4}$ \\ ${ }^{1}$ Department of Urology, Santa Chiara Regional Hospital, Trento 38123; Departments of ${ }^{2}$ Urology, \\ ${ }^{3}$ Medical Genetics, and ${ }^{4}$ Pathology and Oncology, University of Florence, Florence 50121, Italy
}

Received July 31, 2014; Accepted April 17, 2015

DOI: $10.3892 / \mathrm{ol} .2015 .3616$

\begin{abstract}
Loss of heterozygosis ( $\mathrm{LOH})$ on chromosome (Chr) 18q21-23 was reported to be one of the most common genetic alterations identified in bladder cancer. The current study aimed to determine the prognostic role of $\mathrm{LOH}$ on Chr 18q21-23 in patients diagnosed with muscle-invasive urothelial bladder carcinoma (MIBC). A total of 34 consecutive patients were enrolled in the present prospective study. LOH on Chr 18 was assessed by performing multiplex polymerase chain reaction on paired blood and tumour tissue samples from each patient. The following primers were used in the present study: D18S51, MBP LW and MBP H. These data were then compared with follow-up information. The main outcome measure was patient status at the end of the follow-up. Cox regression was used to evaluate the impact of each parameter on cancer-specific survival and the Kaplan Meier test for disease-free survival was plotted in order to estimate survival. Out of 34 patients, 18 (52.9\%) exhibited $\geq 1$ alteration in one of the loci analysed on chromosome 18 , while $16(47.1 \%)$ revealed no alterations. No correlation was identified with stage $(\mathrm{P}=0.18)$ or grade $(\mathrm{P}=0.06)$; however, LOH on Chr 18q21-23 was significantly associated with a lower recurrence-free probability $(\mathrm{P}<0.0001)$. Kaplan-Meier curves demonstrated a significant association between patient status at follow-up and LOH on Chr 18 ( $\mathrm{P}<0.001)$. In addition, multivariate analysis identified $\mathrm{LOH}$ on $\mathrm{Chr} 18(\mathrm{P}<0.001)$ and stage $(\mathrm{P}=0.01)$ as independent survival predictors. Furthermore, artificial neural network analysis was consistent with the results of the multivariate analysis. In conclusion, the present study highlighted the role of LOH on Chr 18q21-23 in predicting the clinical outcome of patients with MIBC.
\end{abstract}

Correspondence to: Professor Tommaso Cai, Department of Urology, Santa Chiara Regional Hospital, Largo Medaglie d'Oro 9, Trento 38123, Italy

E-mail: ktommy@libero.it

Key words: urothelial cancer, invasive bladder cancer, microsatellite analysis, chromosome 18 , loss of heterozygosis

\section{Introduction}

Urothelial carcinoma (UC) of the urinary bladder is a relevant social problem with almost 67,160 novel cases diagnosed each year and accounts for $\sim 13,750$ cancer-associated mortalities each year in the United States (1). Mhawech-Fauceglia et al (2) suggested that the pathology of bladder carcinoma was a difficult to fully elucidate due to the complex oncogenic pathways involved and the inconsistent clinical behaviour of the disease.

Numerous previous studies have identified potential molecular markers for bladder carcinoma in an effort to fully elucidate the cellular mechanisms involved in its pathogenesis and development. Consequently, the prediction of tumour biological potential may help to select patients for treatment, thus improving the survival rates and quality of life of these patients (3-5). Tumour genome analysis has been used to obtain information regarding the natural history of UC (6). Of note, the loss of heterozygosis ( $\mathrm{LOH}$ ) on chromosome (Chr) 18 was reported to be the initial genetic event in bladder cancer (7).

As tumour-suppressor genes, including the DCC (18q21.3) and DPC4 (18q21.1) genes were identified on Chr 18q (8), it was suggested that somatic alterations on this chromosome may be a critical step for bladder carcinogenesis. In addition, it was reported that loss or inactivation of the SMAD4/DPC4 gene may be involved in the onset of various types of cancer, while $\mathrm{LOH}$ of $18 \mathrm{q} 21.1$ was demonstrated to be associated with a poor prognosis in bladder carcinoma patients (8). The distal section of Chr 18 (18q21-q23) was considered to be a potential locus of numerous crucial genes for the pathogenesis and progression of bladder cancer. A previous study highlighted the role of $\mathrm{LOH}$ analysis of $\mathrm{Chr} 18$ for enhancing the prediction of recurrence in patients with low-grade non-muscle-invasive bladder cancer (4); however, further studies are required. Notably, the D18S51, MBP LW and MBP $\mathrm{H}$ loci are located on the long arm of $\mathrm{Chr} 18$, where a number of tumor suppressor genes, including DCC and DPC4, are located (6).

The present study aimed to evaluate $\mathrm{LOH}$ on $\mathrm{Chr} 18$ in patients with muscle-invasive urothelial bladder cell carcinomas in order to determine whether there is an association between $\mathrm{LOH}$ on Chr 18 and tumour stage. In addition, the 
present study aimed to investigate whether $\mathrm{LOH}$ on $\mathrm{Chr} 18$ has a role in predicting the clinical outcome of patients with muscle-invasive urothelial bladder cancer.

\section{Materials and methods}

Study design. LOH on Chr 18 was investigated in muscle-invasive UC of the urinary bladder and the findings were then correlated with patient follow-up data. A total of 34 consecutive patients were recruited for the present prospective study who underwent a transurethral resection of bladder tumour (TURBT) at the Department of Urology, University of Florence (Florence, Italy), between January and December 2002. All patients enrolled in the present study were diagnosed with muscle-invasive urothelial bladder cancer [MIBC; $\geq$ primary tumour stage (pT)2, according to the European Association of Urology guidelines] and were able to comply with the follow-up schedules. Patients were excluded if they had a history of UC of the upper urinary tract, prostate cancer or other urologic cancer; in addition, patients with associated carcinoma in situ were omitted from the present study. All patients with other urologic diseases were also excluded, as were all patients lost to follow-up. All selected patients provided written informed consent and the study was approved by the research ethics committee of the University of Florence. The present study was conducted in accordance with the latest version of the Declaration of Helsinki (2008) and in line with Good Clinical Practice guidelines (9).

Specimen collection, histological and molecular analysis. Blood samples were collected from all patients during clinical evaluation and stored at $-80^{\circ} \mathrm{C}$ until molecular analysis. Fresh tumour tissue samples were obtained during TURBT for pathological evaluation and were snap-frozen in liquid nitrogen in the operating room and stored at $-80^{\circ} \mathrm{C}$ until molecular analysis. Tumour and normal DNA were extracted using the methods previously described (5). Briefly, the DNA of each sample underwent digestion with sodium dodecyl sulfate proteinase K (recombinant; Worthington Biochemical Corporation, Lakewood, $\mathrm{NJ}$, USA; $55^{\circ} \mathrm{C}$ overnight), ribonuclease (Multiplex PCR Kit; Qiagen Spa, Milan, Italy) treatment ( $2 \mathrm{~h}$ at $37^{\circ} \mathrm{C}$ ) and phenol chloroform (Life Technologies Italia, Monza, Italy) extraction. DNA was resuspended in $700 \mathrm{ml}$ Tris EDTA (Life Technologies Italia). The quantity of DNA was evaluated by spectrophotometry optical density (NanoDrop ${ }^{\circledR} 1000 U V$ spectrophotometer; Thermo Fisher Scientific, Inc., Wilmington, DE, USA), and the samples were diluted to a concentration of $4 \mathrm{ng} / \mu 1$ for amplification by polymerase chain reaction (PCR). PCR was performed in a final volume of $15 \mathrm{ml}$ with $10 \mathrm{ng}$ DNA in order to amplify genomic DNA at three specific Chr 18 loci: D18S51, MBP LW and MBP H. The primer sequences were as follows: Forward, 5'-TTCTTGAGC CCAGAAGGTTA-3' and reverse, 5'-ATTCTACCAGCAACA ACACAAATAAAC-3' for D18S51; forward, 5'-TGGCTA CTTGGGCTATTGTAAACG-3' and reverse, 5'-GGTGGT TCTGTTCCCTCTATCTCC-3' for MBP LW; and forward, 5'-TCCGAGCAGCAGCCAGCAC-3' and reverse, 5'-AAG CTCGTCGGACTCTGAG-3' for MBP H. Each primer was fluorescently marked with the dyes 6-FAM, NED and HEX (Applied Biosystems Life Technologies, Foster City, CA,
USA). Normal and pathological DNA underwent amplification in an Applied Biosystems ${ }^{\circledR} 2720$ Thermal Cycler (Life Technologies Italia); three PCRs were performed per patient. The amplification conditions of the reactions were a denaturation cycle at $95^{\circ} \mathrm{C}$ for $10 \mathrm{~min}, 32$ cycles at $95^{\circ} \mathrm{C}$ for $30 \mathrm{sec}, 56^{\circ} \mathrm{C}$ for D18S51 and $52^{\circ} \mathrm{C}$ for MBP LW and MBP H, for $30 \mathrm{sec}$, and $72^{\circ} \mathrm{C}$ for $1 \mathrm{~min}$, and final extension at $72^{\circ} \mathrm{C}$ for $45 \mathrm{~min}$. DNA quality was assessed by agarose gel and ethidium bromide staining (Life Technologies Italia). Amplification products $(1 \mu \mathrm{l})$ were mixed with $12 \mu$ f formamide (molecular biology grade; Life Technologies Italia) and 0.5 1 GeneScan ROX400 (Perkin Elmer Biosystems, Foster City, CA, USA), denatured at $95^{\circ} \mathrm{C}$ for $10 \mathrm{~min}$ and then rapidly iced. Up to 48 samples were then electrophoresed in an ABI Prism ${ }^{\circledR} 310$ Genetic Analyzer (Life Technologies Italia). Amplification products $(50 \mu \mathrm{l})$ were injected electrokinetically in a $47 \mathrm{~cm}$ capillary (Applied Biosystems Life Technologies) filled by performance optimized polymer 4 (Life Technologies Italia). Electrophoresis was conducted at $15 \mathrm{kV}$ for $24 \mathrm{~min}$ at $60^{\circ} \mathrm{C}$ and electrodes were immersed in ABI Prism ${ }^{\circledR} 310$ Genetic Analyzer Buffer (Life Technologies Italia). Numerical data collected during electrophoresis were analyzed by GeneScan software (version 3.1; Thermo Fisher Scientific, Inc.) to produce graphic and numerical results of dimension of amplified DNA fragments. The microsatellite markers employed in the present study and their locations were taken from the Genome Database (http://www.ncbi.nlm.nih.gov/genome). The primers for the MBP gene repeats also amplified two short tandem repeat loci (locus A and locus B) (10). Microsatellite sequences observed to be most frequently altered in previous studies $(6,11,12)$ were selected for the present study. LOH was characterised as the complete or almost complete absence of one allele in tumour DNA. All molecular tests were performed in duplicate using isolated PCR reactions. All available haematoxylin and eosin-stained slides of bladder carcinomas were reviewed by one pathologist. Specimens were pathologically staged according to the 2010 Tumour-Node-Metastasis classification of the American Joint Committee on Cancer (13) and tumour grade was assigned in line with the 2004 World Health Organization/International Society of Urologic Pathology classification (14).

Patients. Following the diagnosis of MIBC, all patients underwent radical cystectomy with standard bilateral pelvic lymphadenectomy and urinary diversion. Radical cystectomy was performed by an expert uro-oncology consultant according to the procedure described by the International Consultation on Bladder Cancer (15). The lymph node dissection performed involved the excision of all lymphatic tissues surrounding the common iliac, external iliac, internal iliac and obturator arteries as well as the presacral region. Following cystectomy and urinary diversion, all patients underwent follow-up examinations, which were performed according to the European Association of Urology guidelines (16). In brief, chest x-rays and abdominal ultrasound were required every 3 months, computerised tomography of the abdomen every 6 months and bone scan and excretory urography every 12 months. Additional examinations were required for symptomatic disease (16). Concomitant urethrectomy was performed only in patients who had preoperative histologically proven UC of the prostate and/or urethra in association with MIBC (16). 
Table I. Summary of clinical and histopathological patient data.

\begin{tabular}{lc} 
A, Patient characteristics & \\
\hline Characteristic & $\mathrm{n}(\%)$ \\
\hline No. of patients & 34 \\
Mean age \pm standard deviation & $69.9 \pm 8.7$ \\
Gender & \\
Male & $32(94.1)$ \\
Female & $2(5.9)$ \\
\hline
\end{tabular}

B, Patient anamnestic, pathological and clinical data

\begin{tabular}{ll}
\hline Characteristic & $\mathrm{n}(\%)$
\end{tabular}

No. of recurrences/year

1

2

$\geq 3$

$9(26.5)$

No. of lesions

1

$21(61.8)$

2

$\geq 3$

6 (17.6)

Diameter of lesion (if multiple,

diameter of the largest)

$<3 \mathrm{~cm}$

24 (70.6)

$\geq 3 \mathrm{~cm}$

Stage

pT2

$19(55.9)$

7 (20.6)

5 (14.7)

pT3b

$3(8.8)$

Grade

G3

34 (100)

Lymph node involvement

N0

$28(82.3)$

N1

$6(17.7)$

$0(0.0)$

$0(0.0)$

N3

Previous intravesical therapy

34 (100)

Cigarette smokers

Charlson comorbidities index

$<2$

$26(76.5)$

2

$6(17.6)$

$\geq 3$

2 (5.9)

Urinary diversion

Cutaneous ureterostomy

$11(32.4)$

6 (17.6)

$17(50.0)$

Neobladder

$15.1(11-23)$

Mean no. of lymph nodes removed (range)

3 (8.8)

pT, primary tumour stage; G, grade; N, node involvement.

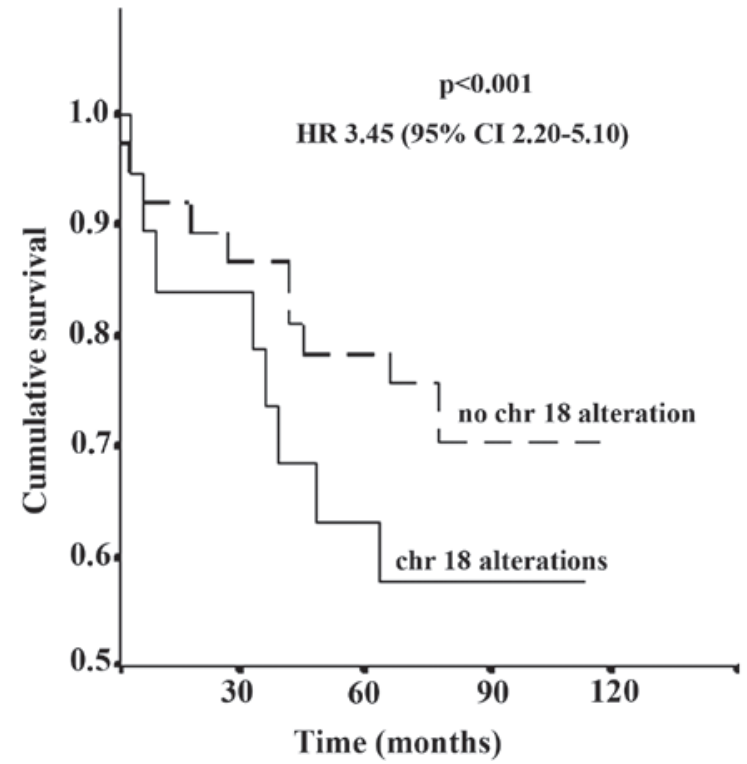

Figure 1. Kaplan-Meier curves illustrating the association between LOH on chr 18 (HR, 3.45; 95\% CI, 2.20-5.10; P<0.001) and cancer-specific survival patient probability. Mortality was used as endpoint in Kaplan-Meier analysis. Time (months), time from initial diagnosis to first recurrence (months); LOH, loss of heterozygosis; chr, chromosome; HR, hazard ratio; CI, confidence interval.

Statistical analysis. As the null hypothesis, $\mathrm{LOH}$ on $\mathrm{Chr} 18$ was assumed to have no impact on survival rate in patients with MIBC. The significance of all parameters was assessed using the Fisher's exact test and chi-square test, where $\mathrm{P}<0.05$ was considered to indicate a significant difference between values. The $95 \%$ confidence intervals (CIs) were calculated for the probability of survival for the Kaplan-Meier estimates. Mann-Whitney tests were also used in order to compare the means of the different parameters. Univariate and multivariate relative risk was calculated using Cox proportional hazards regression analysis. SPSS 11.5 for Apple-Macintosh (SPSS, Inc., Chicago, IL, USA) was used to perform all statistical analyses.

Artificial Neural Network (ANN) analysis was employed in order to enhance the standard statistical analysis, as previously described $(17,18)$. In brief, a neural network was used in order to predict the outcome of patients with MIBC, according to the findings of the univariate and multivariate analyses. The following factors were the input parameters (input neuron) for ANN analysis: Age, gender, number of lesions, diameter of lesions, $\mathrm{LOH}$ on $\mathrm{Chr}$ 18, stage and grade. ANN setup was performed using the commercially available NeuralWorks Predict software (2005; NeuralWare Inc., Carnegie, PA, USA).

\section{Results}

Patient and tumour characteristics. A total of 34 patients were enrolled in the present study, 32 of which were male and 2 were female (mean age, 69.9 \pm 8.7 ). The patient and tumour characteristics are detailed in Table I.

Molecular results. At the baseline, 18 (52.9\%) patients exhibited $\geq 1$ alteration in one of the loci analysed on Chr 18 , while $16(47.1 \%)$ showed no alterations on Chr 18. Out of the 
18 patients with altered loci, the MBP LW locus was altered in 6 (33.3\%), MBP H in 7 (38.9\%) and D18S51 in $5(27.8 \%)$ patients. In 2 patients the D18S51 locus was singularly altered, in 1 patient alterations occurred on both MBP $\mathrm{H}$ and D18S51 loci and 2 patients exhibited altered MBP LW and D18S51 loci.

Follow-up results. At a mean follow-up duration of 128.6 months post-surgery, 11 patients (32.3\%) were alive with no evidence of disease (mean disease-free survival time, 126.6 months; 95\% CI, 119.2-129.1 months). In addition, 8 patients were alive with evidence of tumour recurrence (mean disease-free survival time, 10.8 months; $95 \%$ CI, 103.5-115.1 months) and a further 11 patients succumbed to their disease (mean disease-free survival time, 23.2 months; 95\% CI, 19.7-29.1 months) as well as four mortalities from non-associated causes. Cumulative cancer-specific survival rates of the whole study group at 1,3 and 5 years were 88.2, 64.7 and $52.9 \%$, respectively. No correlation was identified between clinical or pathological factors and the detection of LOH on Chr 18. Kaplan-Meier analysis revealed a significant correlation between patient status at follow-up and LOH on Chr 18 [hazard ratio (HR), 3.45; 95\% CI, 2.20-5.10; P<0.001] (Fig. 1). The comparison of microsatellite analysis with follow-up data identified significant correlations between altered D18S51 $(\mathrm{P}<0.001)$ or MBP H (HR, 2.61; 95\% CI, 1.80-3.12; P<0.001) loci and patient status at follow-up. However, no significant correlation was demonstrated between MBP LW and status at follow-up $(\mathrm{P}=0.6)$.

Univariate analysis, multivariate analysis and artificial ANN results. As determined using univariate analysis, stage, lymph node status, $\mathrm{LOH}$ on $\mathrm{Chr} 18$ and age proved to be significantly associated with patient survival. According to the multivariate analysis, the detection of $\mathrm{LOH}$ on $\mathrm{Chr} 18$ (HR, 4.08; 95\% CI, 2.09-6.61; $\mathrm{P}=0.001)$ and stage $(\geq \mathrm{pT} 2$; HR, 2.23; 95\% CI, 1.17-3.46; $\mathrm{P}=0.01$ ) were identified as independent prognostic factors in predicting status at follow-up. ANN analysis identified $\mathrm{LOH}$ on $\mathrm{Chr} 18$ and stage as the most powerful variables affecting the output decision and predicting the natural history of MIBC; these results were consistent with those obtained by multivariate analysis.

\section{Discussion}

Optimal control and management of patients with bladder cancer is primarily dependent on appropriate risk-group stratification, which is established according to the correct assessment of biological and clinical characteristics (19). Numerous previous studies have identified several sequential genetic events, which were found to be associated with tumour natural history (8). A novel bladder cancer susceptibility locus, the urea transporter gene SLC14A1 was identified on Chr 18q $(20,21)$. In addition, $\mathrm{LOH}$ on Chr 18q and 9q was reported to be able to predict the clinical outcome of patients with bladder cancer (8).

The present study demonstrated that $\mathrm{LOH}$ on $\mathrm{Chr} 18$ was an independent prognostic indicator of survival in MIBC patients. Three loci were evaluated on the $\mathrm{q}$ arm of $\mathrm{Chr} 18$ (18q21-23), where two tumour-suppressor genes, DCC (18q21.3) and DPC4 (18q21.1), are located (8). Uchida et al (8) suggested that Chr 18q was crucial in the development of the malignant phenotype, particularly in bladder cancer $(7,8)$. Of note, in the present study, a strong correlation was observed between altered D18S51 or MBP H loci and patient outcome. A previous study, which investigated non-muscle-invasive UC, reported no notable correlation between D18S51 locus and status at follow-up (4), emphasising that $\mathrm{LOH}$ on $\mathrm{Chr} 18 \mathrm{q} 22$ (D18S51 locus) may be a late event in bladder tumorigenesis (22). Brewster et al (22) demonstrated that loss of genetic material on 18q21.3, which includes DCC, was associated with MIBC disease and was frequently present in tumour recurrences. The results of the present study confirmed that alterations in MBP LW and MBP H loci occurred in the early phase of bladder tumorigenesis, while the D18S51 locus was implicated in a later phase. Therefore, alteration in the D18S51 locus identified on tissue samples following TURBT may be considered as a negative prognostic factor affecting survival in patients with MIBC. A limitation of the present study is the small number of the patients analysed, this was due to the time-consuming and labour-intensive, thus expensive, molecular biology techniques employed. To the best of our knowledge, the current study was the first to demonstrate the use of $\mathrm{LOH}$ on $\mathrm{Chr} 18 \mathrm{q} 21-23$ in predicting the clinical outcome of patients with MIBC; however, further studies are required in order to confirm the present findings.

In conclusion, the present study highlighted the role of LOH on Chr 18q21-23 in predicting the clinical outcome of patients with MIBC. In addition, the present study elucidated the feasibility and utility of $\mathrm{Chr} 18 \mathrm{LOH}$ as a potential clinical marker, in conjunction with well-established clinico-pathological factors, for the development of an effective adjuvant treatment schedule in MIBC patients.

\section{Acknowledgements}

The authors would like to thank Professor John Denton, Department of Modern Philology, University of Florence (Florence, Italy) for manuscript language revision.

\section{References}

1. Jemal A, Siegel R, Ward E, et al: Cancer statistics, 2007. CA Cancer J Clin 57: 43-66, 2007.

2. Mhawech-Fauceglia P, Cheney RT and Schwaller J: Genetic alterations in urothelial bladder carcinoma: An updated review. Cancer 106: 1205-1216, 2006.

3. Tomasini JM and Konety BR: Urinary markers/cytology: What and when should a urologist use. Urol Clin North Am 40: 165-173, 2013.

4. Cai T, Nesi G, Dal Canto M, Mondaini N, Piazzini M and Bartoletti R: Prognostic role of loss of heterozygosity on chromosome 18 in patients with low-risk nonmuscle-invasive bladder cancer: Results from a prospective study. J Surg Res 161: 89-94, 2010.

5. Bartoletti R, Cai T, Nesi G, Roberta Girardi L, Baroni G and Dal Canto M: Loss of P16 expression and chromosome 9p21 $\mathrm{LOH}$ in predicting outcome of patients affected by superficial bladder cancer. J Surg Res 143: 422-427, 2007.

6. Bartoletti R, Cai T, Dal Canto M, Boddi V, Nesi G and Piazzini M: Multiplex polymerase chain reaction for microsatellite analysis of urine sediment cells: A rapid and inexpensive method for diagnosing and monitoring superficial transitional bladder cell carcinoma. J Urol 175: 2032-2037, 2006.

7. Knowles MA: What we could do now: Molecular pathology of bladder cancer. Mol Pathol 54: 215-221, 2001. 
8. Uchida A, Tachibana M, Miyakawa A, Nakamura K and Murai M: Microsatellite analysis in multiple chromosomal regions as a prognostic indicator of primary bladder cancer. Urol Res 28: 297-303, 2000.

9. Switula D: Principles of good clinical practice $(\mathrm{GCP})$ in clinical research. Sci Eng Ethics 6: 71-77, 2000.

10. Polymeropoulos MH, Xiao H and Merril CR: Tetranucleotide repeat polymorphism at the human myelin basic protein gene (MBP). Hum Mol Genet 1: 658, 1992.

11. Bartoletti R, Dal Canto M, Cai T, Piazzini M, Travaglini F, Gavazzi A and Rizzo M: Early diagnosis and monitoring of superficial transitional cell carcinoma by microsatellite analysis on urine sediment. Oncol Rep 13: 531-537, 2005.

12. Dal Canto M, Bartoletti R, Travaglini F, Piazzini M, Lodovichi G, Rizzo M and Selli C: Molecular urinary sediment analysis in patients with transitional cell bladder carcinoma. Anticancer Res 23: 5095-5100, 2003.

13. Edge SB, Byrd DR, Compton CC, et al (eds): American Joint Committee on Cancer Staging Manual. 7th edition. Springer, New York, NY, pp117-126, 2010.

14. Eble JN, Sauter G,Epstein JI and Sesterhenn IA (eds): World Health Organization Classification of Tumours: Pathology and Genetics of Tumours of the Urinary System and Male Genital Organs. IARC Press, Lyon, France, pp89-154, 2004.

15. Gakis G, Efstathiou J, Lerner SP, et al; International Consultation on Urologic Disease-European Association of Urology Consultation on Bladder Cancer 2012: ICUD-EAU International Consultation on Bladder Cancer 2012: Radical cystectomy and bladder preservation for muscle-invasive urothelial carcinoma of the bladder. Eur Urol 63: 45-57, 2013
16. Stenzl A, Cowan NC, De Santis M, Kuczyk MA, Merseburger AS, Ribal MJ, Sherif A and Witjes JA; European Association of Urology (EAU): Treatment of muscle-invasive and metastatic bladder cancer: Update of the EAU guidelines. Eur Urol 59: 1009-1018, 2011.

17. Cai T, Conti G, Lorenzini M and Bartoletti R: Artificial intelligences in urological practice: The key to success? Ann Oncol 18: 604, 2007.

18. Cai T, Conti G, Nesi G, et al: Artificial intelligence for predicting recurrence-free probability of noninvasive high-grade urothelial bladder cell carcinoma. Oncol Rep 18: 959-964, 2007.

19. Lee SE and Park MS: Prognostic factors for survival in patients with transitional cell carcinoma of the bladder: Evaluation by histopathologic grade, pathologic stage and flow-cytometric analysis. Eur Urol 29: 193-198, 1996.

20. Garcia-Closas M, Ye Y, Rothman N, Figueroa JD, Malats N, Dinney CP, Chatterjee N, Prokunina-Olsson L, Wang Z, Lin J et al: A genome-wide association study of bladder cancer identifies a new susceptibility locus within SLC14A1, a urea transporter gene on chromosome 18q12.3. Hum Mol Genet 20: 4282-4289, 2011.

21. Koutros S, Baris D, Fischer A, et al: Differential urinary specific gravity as a molecular phenotype of the bladder cancer genetic association in the urea transporter gene, SLC14A1. Int J Cancer 133: 3008-3013, 2013.

22. Brewster SF, Gingell JC, Browne S and Brown KW: Loss of heterozygosity on chromosome $18 \mathrm{q}$ is associated with muscle-invasive transitional cell carcinoma of the bladder. Br J Cancer 70: 697-700, 1994. 